STUDI

FRANCESI

\section{Studi Francesi}

Rivista quadrimestrale fondata da Franco Simone

165 (LV | III) | 2011

LA RAPPRESENTAZIONE DELLA MADRE NELLA

LETTERATURA FRANCESE DEL NOVECENTO a cura di Dario Cecchetti e Michele Mastroianni

\title{
Autour de Bernardin de Saint-Pierre: les écrits et les hommes des Lumières à l'Empire, études recueillies par Catriona Seth et Éric Wauters
}

\section{Vittorio Fortunati}

\section{(2) OpenEdition}

\section{Journals}

\section{Edizione digitale}

URL: http://journals.openedition.org/studifrancesi/5026

DOI: 10.4000/studifrancesi.5026

ISSN: 2421-5856

\section{Editore}

Rosenberg \& Sellier

\section{Edizione cartacea}

Data di pubblicazione: 1 décembre 2011

Paginazione: 645-646

ISSN: 0039-2944

\section{Notizia bibliografica digitale}

Vittorio Fortunati, «Autour de Bernardin de Saint-Pierre: les écrits et les hommes des Lumières à l'Empire, études recueillies par Catriona Seth et Éric Wauters», Studi Francesi [Online], 165 (LV | III) | 2011, online dal 30 novembre 2015, consultato il 08 janvier 2021. URL: http://journals.openedition.org/ studifrancesi/5026 ; DOI: https://doi.org/10.4000/studifrancesi.5026

Questo documento è stato generato automaticamente il 8 janvier 2021.

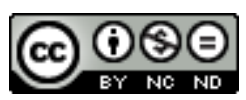

Studi Francesi è distribuita con Licenza Creative Commons Attribuzione - Non commerciale - Non opere derivate 4.0 Internazionale. 


\title{
Autour de Bernardin de Saint-Pierre: les écrits et les hommes des Lumières à l'Empire, études recueillies par Catriona Seth et Éric Wauters
}

\author{
Vittorio Fortunati
}

\section{NOTIZIA}

Autour de Bernardin de Saint-Pierre: les écrits et les hommes des Lumières à l'Empire, études recueillies par Catriona SETH et Éric WAUTERS, Publications des Universités de Rouen et du Havre, 2010, pp. 228.

1 Sebbene Bernardin de Saint-Pierre sia stato uno degli autori francesi più letti negli ultimi due secoli (grazie soprattutto, s'intende, a Paul et Virginie), le ricerche a lui dedicate hanno segnato a lungo il passo, se così si può dire: lo prova anche la mancanza, nel momento in cui redigiamo queste note, di un'edizione moderna delle sue opere complete. Solo recentemente si è assistito a un rinnovarsi dell'interesse degli studiosi, che si è concretizzato, fra l'altro, nel colloquio internazionale tenutosi a Rouen e a Le Havre dal 26 al 28 ottobre 2006, i cui atti hanno visto finalmente la luce.

2 Il volume si apre con una sezione incentrata sulle relazioni epistolari e mondane di Bernardin: il contributo di Simon DAvies (Pierre-Michel Hennin: le correspondant le plus fidèle de Bernardin de Saint-Pierre, pp. 13-19) riguarda le numerose lettere scambiate dallo scrittore con un diplomatico appassionato di letteratura; quello di Philip RoBINSON (Les lettres de Mme Poivre ne sont pas un roman, pp.21-29) rievoca l'intima amicizia di Bernardin con la moglie del governatore dellîle de France; Irène PASSERAN (Liberté, vérité, pauvreté: Bernardin de Saint-Pierre chez d'Alembert et Mlle de Lespinasse, pp. 31-51) segue lo scrittore nella sua frequentazione dei salons parigini; la corrispondenza con Marion Delaville-Jehannin e con Madeleine-Sophie Challe è al centro del contributo di 
Tim REEVE (De retour de l'île de France: la sympathie des femmes dans des temps incertains, pp. 53-57); Rebecca FORD (Une correspondance amicale: Bernardin de Saint-Pierre et Mesnard de Conichard, pp.59-66) evidenzia il ruolo di un personaggio ancora poco conosciuto nella vita e nella carriera letteraria di Bernardin de Saint-Pierre.

Nei tre articoli che constituiscono la seconda sezione del volume, l'attenzione degli studiosi si concentra sugli anni a ridosso dello scoppio della Rivoluzione: Malcolm cook (Une année dans la vie de Bernardin de Saint-Pierre: 1788, pp.69-77) mostra come la pubblicazione di Paul et Virginie abbia cambiato radicalmente l'esistenza del suo autore; Branko ALEKSIC' (Casanova examine les "Études de la nature" et "Paul et Virginie" de Bernardin de Saint-Pierre en 1788, pp. 79-89) propone un interessante parallelo tra il romanziere francese e il colto avventuriero italiano; Katherine ASTBURY (La Révolution au féminin: les lettres de Catherine de Saint-Pierre à l'époque révolutionnaire, pp. 91-99) analizza le lettere della sorella di Bernardin, che descrivono bene i primi moti rivoluzionari nella città di Dieppe.

4 La terza sezione è la più corposa e raccoglie una serie di originali interpretazioni e di stimolanti letture intertestuali. Alain GUYOT ripercorre il processo di elaborazione del Voyage à l'île de France (1773) a partire dalle lettere dell'autore (Le voyage à l'île de France: des lettres au livre, pp. 103-114). Robin HOWELLs rivela come Bernardin inauguri un nuovo modo di descrivere la natura che anticipa, entro certi limiti, la «forêt de symboles» di Baudelaire (Formes et sensations: les "correspondances" de la nature dans "Paul et Virginie", pp. 115-128). Due opere liriche messe in scena durante la Rivoluzione (Paul et Virginie di Kreutzer, Paulin et Virginie di Le Sueur) sono oggetto del contributo di Patrick TAÏEB ( $L a$ réception de "Paul et Virginie" à travers deux adaptations lyriques pendant la Révolution, pp. 129-140). Secondo Gabriel-Robert тнIBAULt, la formazione scientifica di Bernardin ha il suo compimento nell'elaborazione di una teologia (Science de l'ingénieur et théologie naturelle dans l'ouvre de Bernardin de Saint-Pierre, pp.141-156). Per Colas Duflo, l'osservazione della natura è alla base, nel pensiero dello scrittore, non solo di un'etica, ma anche di un'estetica (Le finalisme esthétisant des "Études de la nature" de Bernardin de Saint-Pierre, pp.157-163). Jean-Michel RACAULT mette in rilievo le connessioni fra l'esaltazione della vita ritirata e la critica delle ingiustizie sociali (Le Solitaire et les "corps": l'imaginaire politique de Bernardin de Saint-Pierre et la fin de l'Ancien Régime, pp.166-186). Sophie LEFAY inserisce le riflessioni dello scrittore sulla funzione pedagogica dei monumenti nel quadro dei dibattiti della sua epoca (La voix des pierres: Bernardin de Saint-Pierre et le goût des inscriptions, pp. 187-197). Infine, Cristina ROMANo (L'influence de Bernardin de Saint-Pierre dans les premières ceuvres de Chateaubriand, pp.199-213) sottolinea come il giovane René, oltre a condividere la sensibilità di Bernardin per il fascino delle rovine, ne sia stato influenzato nel modo di rappresentare la natura. 